\title{
Aggression subtypes relate to distinct resting state functional connectivity in children and adolescents with disruptive behavior
}

\author{
Julia E. Werhahn ${ }^{1,2}$ (1) - Susanna Mohl ${ }^{1} \cdot$ David Willinger $^{1,2} \cdot$ Lukasz Smigielski $^{1} \cdot$ Alexander Roth $^{1,2}$. \\ Christoph Hofstetter ${ }^{1}$. Philipp Stämpfli ${ }^{3}$. Jilly Naaijen ${ }^{4,5}$. Leandra M. Mulder ${ }^{4,5}$. Jeffrey C. Glennon ${ }^{4}$. \\ Pieter J. Hoekstra ${ }^{6} \cdot$ Andrea Dietrich $^{6} \cdot$ Renee Kleine Deters $^{6} \cdot$ Pascal M. Aggensteiner $^{7} \cdot$ Nathalie E. Holz $^{7}$. \\ Sarah Baumeister ${ }^{7}$. Tobias Banaschewski ${ }^{7}$. Melanie C. Saam ${ }^{8}$. Ulrike M. E. Schulze ${ }^{8}$. David J. Lythgoe ${ }^{9}$. \\ Arjun Sethi ${ }^{10}$. Michael C. Craig ${ }^{10} \cdot$ Mathilde Mastroianni $^{11} \cdot$ Ilyas Sagar-Ouriaghli ${ }^{11}$ - Paramala J. Santosh ${ }^{11}$. \\ Mireia Rosa ${ }^{12}$. Nuria Bargallo ${ }^{13}$. Josefina Castro-Fornieles ${ }^{14} \cdot$ Celso Arango $^{15} \cdot$ Maria J. Penzol $^{15} \cdot$ Marcel P. Zwiers $^{5}$. \\ Barbara Franke $^{16,17}$. Jan K. Buitelaar ${ }^{4,18}$. Susanne Walitza ${ }^{1,2}$. Daniel Brandeis ${ }^{1,2,7}$
}

Received: 16 November 2019 / Accepted: 8 July 2020 / Published online: 13 August 2020

(c) The Author(s) 2020, corrected publication 2020

\begin{abstract}
There is increasing evidence for altered brain resting state functional connectivity in adolescents with disruptive behavior. While a considerable body of behavioral research points to differences between reactive and proactive aggression, it remains unknown whether these two subtypes have dissociable effects on connectivity. Additionally, callous-unemotional traits are important specifiers in subtyping aggressive behavior along the affective dimension. Accordingly, we examined associations between two aggression subtypes along with callous-unemotional traits using a seed-to-voxel approach. Six functionally relevant seeds were selected to probe the salience and the default mode network, based on their presumed role in aggression. The resting state sequence was acquired from 207 children and adolescents of both sexes [mean age (standard deviation) $=13.30$ (2.60); range $=8.02-18.35]$ as part of a Europe-based multi-center study. One hundred eighteen individuals exhibiting disruptive behavior (conduct disorder/oppositional defiant disorder) with varying comorbid attention-deficit/hyperactivity disorder (ADHD) symptoms were studied, together with 89 healthy controls. Proactive aggression was associated with increased left amygdala-precuneus coupling, while reactive aggression related to hyper-connectivities of the posterior cingulate cortex (PCC) to the parahippocampus, the left amygdala to the precuneus and to hypo-connectivity between the right anterior insula and the nucleus caudate. Callous-unemotional traits were linked to distinct hyper-connectivities to frontal, parietal, and cingulate areas. Additionally, compared to controls, cases demonstrated reduced connectivity of the PCC and left anterior insula to left frontal areas, the latter only when controlling for ADHD scores. Taken together, this study revealed aggressionsubtype-specific patterns involving areas associated with emotion, empathy, morality, and cognitive control.
\end{abstract}

Keywords Reactive aggression · Proactive aggression · Callous-unemotional traits · Default mode network · Amygdala · Functional connectivity

\section{Introduction}

Electronic supplementary material The online version of this article (https://doi.org/10.1007/s00787-020-01601-9) contains supplementary material, which is available to authorized users.

Julia E. Werhahn

julia.werhahn@gmx.ch

$\triangle$ Daniel Brandeis

brandeis@kjpd.uzh.ch

Extended author information available on the last page of the article
Aggression is defined as behavior aimed to harm other persons or objects. In young individuals, aggression dimensions partially undercut the normative diagnostic categories [1]. Two clinical manifestations within the disruptive behavior disorder (understood as broad hostile and defiant behaviors), oppositional defiant disorder, and conduct disorder are among the most common psychiatric disorders in childhood and adolescence [2]. They are defined by angry and vindictive behaviors and violating rules, norms, and rights, 
respectively [3]. Additionally, callous-unemotional traits are reported in a significant percentage of children and adolescents with disruptive behavior disorder [4] and are characterized by callousness, uncaring, and unemotional dimensions $[5,6]$. Within this complexity of phenotypic manifestation in child and adolescent psychopathology, reactive and proactive aggression (RA and PA, respectively) pertain to distinct functions of aggression, reflecting impulsive and instrumental behaviors, accordingly [7]. On the neural level, differentiation between these two subtypes in humans remains largely unexplored. Yet, there is substantial evidence from animal models and research on neurotransmitters that both forms tap into distinct neural circuits and are linked to acute responses to a threat (RA) or a self-initiated, predatory action (PA) [8].

Among human neuroimaging paradigms, the investigation of brain activity during a resting state [9] has the potential to penetrate clinical practice [10]. It seems especially well-suited to enhance our understanding of the neural foundation of disruptive behavior in children and adolescents. The procedure can be performed in individuals unable to cooperate during cognitive tasks. It targets spontaneous brain activity and delivers biological metrics, such as functional connectivity, which was found to identify inter-individual differences $[11,12]$. For example, recent functional magnetic resonance imaging (fMRI) studies of resting state in conduct disorder have reported both increases and decreases in functional connectivity or activity depending on the network. Specifically, the brain regions involved included the amygdala and insula, parts of the salience network (SN) [13-15], as well as the default mode network (DMN) $[16,17]$. So far, only a few resting state studies have evaluated callous-unemotional traits. Decoupling between the DMN and fronto-parietal network was found to increase with expression of callous-unemotional traits, indicative of dissociation between cognitive control and a social understanding of others [18].

On the other hand, within-network DMN connectivity was shown to increase with higher callous-unemotional traits, suggesting alterations in self-referential processing [19]. Moreover, the interpersonal/affective dimensions were found to depend on DMN connectivity [20]. Additionally, in male youths with conduct disorder, interpersonal traits were correlated with distinct amygdala subregional connectivity with regions located in the SN and DMN [14]. Compared to male youths with conduct disorder who scored low on callous-unemotional traits or healthy controls, juveniles with conduct disorder who scored high on callous-unemotional traits showed increased amygdala connectivity with a frontal section of the DMN [15]. The findings mentioned above suggest that brain connectivity involving these two networks may reflect the clinical manifestation.
Comorbid attention-deficit/hyperactivity disorder (ADHD) symptoms are frequently present in conduct problems [21], associated with poorer responses to treatments [22], and linked to overlapping neural deficits in prefrontal and limbic areas [23]. The severity of ADHD symptoms was also linked to enhanced functional density [24] and other alterations $[25,18,26]$ involving the DMN. Previously, the connectivity between core DMN regions, i.e., the anterior medial prefrontal cortex (amPFC) and the posterior cingulate cortex (PCC), was found to be reduced in male adolescents with conduct disorder compared to healthy controls after controlling for ADHD symptoms, as ADHD symptoms were positively correlated with the connectivity of the DMN [17]. Nevertheless, these fMRI studies largely neglected distinct manifestations of aggression [7]. Despite considerable behavioral research accentuating the differentiation between RA and PA-with evidence for both being correlated [27], but also associated with different behavioral symptoms [28] —no investigation of resting state functional connectivity to date has addressed their underlying mechanisms in children and adolescents with disruptive behavior. Additionally, most of the studies have not included healthy controls and discarded additional aggression dimensions.

In consideration of the above rationale, this study examines the distinct patterns of resting state functional connectivity associated with RA and PA, along with callous-unemotional traits, in children and adolescents with disruptive behavior. We applied a well-established seed-tovoxel approach, which computes cross-correlations within time-series data derived from blood-oxygen level dependent (BOLD) signals in a specific seed with the rest of the brain. This approach enables the detection of functional connections with any voxel or cluster of voxels lying within or outside of any specific network. Based on the findings of the altered connectivity of brain areas in the DMN [16, 17, 29, $24]$ and $S N$ [14, 15], we defined the functionally relevant regions of interest to probe these two networks, i.e., within the PCC, amPFC, bilateral anterior insula, and bilateral amygdala. Our hypothesis assumed distinct connectivity patterns for proactive/reactive aggression and callous-unemotional traits $[14,15,19]$. To replicate earlier findings on case-control differences, we expected to observe reduced connectivity in aggressive cases compared to healthy controls and increased connectivity with higher ADHD scores $[17,24]$. Overall, we hypothesized that cases would differ in connectivity and correlations with clinical symptoms, with the involvement of areas linked to the processing of emotion, empathy abilities, and cognitive control, such as the PCC - the prefrontal cortex, amygdala-precuneus, the anterior insula-frontal areas, and/or limbic areas. 


\section{Methods and materials}

\section{Participants}

The participants in the current study were part of the joint EU-MATRICS (https://matrics-project.eu) and EU-Aggressotype (https://www.aggressotype.eu) projects. Both multicenter research initiatives aim to gain new insights into the mechanisms underlying aggression, especially by identifying biological and behavioral correlates for subtypes of aggression. The applied methods include animal models, genomics, epigenomics, transcriptomics, neurochemistry, and human neuroimaging. Children and adolescents aged 8-18 years were recruited from resident hospitals, ambulatories, and eligible (boarding) schools. A total of 207 individuals $(n=150$ males) comprising cases $(n=118)$ and healthy controls $(n=89)$ were included from nine different sites in Europe (mean age \pm standard deviation (SD): $13.30 \pm 2.60$ years). As the main goal was to conduct aggression subtype-specific analyses, recruitment focused on including cases presenting with a diagnosis of conduct disorder and/or oppositional defiant disorder and/or aggression scores in a clinical range ( $T>70$ ) according to the Child Behavior Checklist (CBCL), Youth Self Report (YSR), or Teacher Report Form (TRF) [30]. Cases were required additionally to take no medication or be on stable medication for at least 2 months. Further exclusion criteria were as follows: a primary DSM-5 diagnosis of depression, anxiety, psychosis, or bipolar disorder for cases, and a DSM-5 diagnosis or clinically relevant scores in the CBCL, YSR, or TRF for healthy controls. Additional exclusion criteria for all participants included the following: standard contraindications for MRI scanning (i.e., braces, metal medical implants), an anxiety score $>8$ on a Visual Analogue Scale ranging from 1 to 10 (because anxiety caused by the scanner could impair data quality and alter neural activity [31]), and an IQ score lower than 80. All individuals had sufficient native language skills based on the country of the assessment. Participants and their parents or legal representatives gave written informed consent. Each site obtained ethical approval separately.

\section{Clinical assessments}

The semi-structured interview Kiddie Schedule for Affective Disorders and Schizophrenia Present and Lifetime version (K-SADS-PL) [32] was used to assess the diagnostic criteria for all participants, as conducted by trained psychologists or trained and supervised interns based on the reports of participants and their parents, who were interviewed separately. The self-reported Reactive Proactive Aggression Questionnaire [33] measured the RA and PA forms of aggression. To assess callous-unemotional traits, the parents filled out the Inventory of Callous-Unemotional traits (ICU) [5, 34], which consists of three subscales assessing callousness, uncaring, and unemotional behaviors. ADHD symptoms were evaluated using the inattention, hyperactivity, and impulsivity counts of the K-SADS-PL instrument. Further details on the clinical assessment are provided in the Supplemental Information.

\section{Image acquisition}

For data acquisition, six sites used Siemens $3 \mathrm{~T}(\mathrm{~T})$ scanners, two sites used Philips 3 T scanners, and one site used a GE 3 T scanner (see Supplemental Table S1 and Table S2 for detailed scanner specifications). T1-weighted anatomical scans and $\mathrm{T} 2 *$-weighted echo-planar resting state functional imaging were performed with predominantly similar parameters across sites (TR $2.45 \mathrm{~s}$ or less, at least 32 slices). The average acquisition time was $8 \mathrm{~min} 25 \mathrm{~s}$. The participants were instructed in a standardized fashion to lie still, look at a white crosshair presented against a black background, not think about anything specific, and let their mind wander (to avoid constraining spontaneous thoughts [35]).

\section{Data preprocessing}

Preprocessing was conducted using SPM12 (Welcome Trust Centre for Neuroimaging, UCL, United Kingdom; https:// www.fil.ion.ucl.ac.uk/spm) and the SPM-based CONN toolbox v17.b (https://www.nitrc.org/projects/conn). The initial steps included realignment, unwarping, and slice timing correction. Subsequently, the multi-echo data were linearly weighted by their echo time (TE) using MATLAB (The MathWorks, MA, USA): $Y_{4}=\left(Y_{1} \times \mathrm{TE}_{1} / \mathrm{TE}_{\text {sum }}\right)+\left(Y_{2} \times \mathrm{TE}_{2} /\right.$ $\left.\mathrm{TE}_{\text {sum }}\right)+\left(Y_{3} \times \mathrm{TE}_{3} / \mathrm{TE}_{\text {sum }}\right)$, where $Y$ is the echo file and TE is the echo time. Data were further normalized based on the Montreal Neurological Institute (MNI) brain template [36], followed by an artifact-detection step using the Artifact Detection Tools (ART-toolbox, https://www.nitrc.org/proje cts/artifact_detect), smoothing with a 6-mm full-width at half-maximum Gaussian kernel, and segmentation to derive the white matter and cerebrospinal fluid parameters. The aCompCor strategy [37] implemented in CONN was applied during denoising to reduce the effect of physiological and motion-related noise [38]. Specifically, after identifying principal components of the subject-specific white matter and cerebrospinal confounds, aCompCor extracted the estimated time-series by adding them as regressors. Additionally, the movement parameters derived from realignment were added as regressors. After denoising, the initial hemodynamic response function signal aberrations were removed. Compared to the global signal regression method, aCompCor shows higher sensitivity and specificity regarding positive correlations and anticorrelations [39]. To circumvent 
the influence of low-frequency drifts and high-frequency noise, including heart rate and respiration [38], we applied temporal band-pass filtering $(0.008-0.9 \mathrm{~Hz})$.

\section{Motion censoring}

Given that head motion can lead to changes in BOLD signals [40] and that our cases presenting with externalizing disorders included comorbid ADHD symptoms, we used the threshold for excessive motion applied in recent fMRI studies of adolescents with ADHD [41]. This approach led to the exclusion of ten cases with a root mean square framewise displacement (RMS-FD) of $>0.95 \mathrm{~mm}$. Moreover, a very conservative threshold for detecting functional outlier scans was applied ( $>3 \mathrm{~mm}$ standard deviations from the observed global BOLD signal and $>0.5 \mathrm{~mm}$ composite scan-to-scan motion). Twelve participants were excluded because of the missing or insufficient quality of structural scans, and 14 more were excluded based on image artifacts. Additional sensitivity analyses implementing even stricter motion censoring are provided in the Supplemental Information.

\section{Regions of interest}

Six seeds were chosen, based on their functional relevance for aggression, to probe the DMN and SN [42]. The PCC and amPFC seeds (each including 257 voxels) were created in the MarsBar toolbox (v0.44) (https://marsbar.sourceforg e.net) and centered on coordinates recently used [17] and provided by Andrews-Hanna et al. in their (functional) study of the DMN [43]. The bilateral amygdala and bilateral anterior insula (including 92 and 118 voxels per seed, respectively) are the activation regions of interest derived from the Face Matching Task (negative faces versus shapes) applied and published previously [44]. Given the typically differing connectivities between various parts of the insula and its involvement in emotional processing [45], only the anterior section was selected. Task-related seeds were also chosen to allow future comparisons between the resting state and task within the same study.

\section{Functional connectivity analysis}

Seed-based connectivity analyses were performed using the CONN toolbox. First-level analysis computed Pearson's correlation coefficients between the time course of previously denoised BOLD-signals from seed and whole-brain voxels. After Fisher's transformation to normally distributed $z$-scores, general linear model (GLM) analyses were conducted. Besides site, added as a dummy-coded covariate of no interest in second-level analyses, age, sex, IQ, medication, and handedness were also controlled for based on previous reports of their possible influences on the connectivity of the DMN [46]. A random effects analysis of covariance for group comparisons included further analyses, adding ADHD symptoms as additional covariates of no interest, based on previous reports on the importance of considering ADHD symptoms to differentiate connectivity patterns between cases and controls [17, 26]. Supplemental sensitivity analyses were performed, excluding cases that did not meet the DSM-based diagnosis. Linear regressions separately tested the association between RA, PA, and ICU scores with connectivity values in cases. Supplemental exploratory dimensional analyses (limited by the small and unmatched subsamples) were also performed, in consideration of the putative role of age and sex. The results of the seed-based analyses are reported at thresholds of $p<0.001$ and cluster-corrected $p$-FWE $<0.008(=0.05 / 6$, using additional Bonferroni corrections for the number of seeds).

\section{Results}

\section{Sample characteristics}

Out of 118 cases, 48 had a diagnosis of oppositional defiant disorder, 25 of conduct disorder plus oppositional defiant disorder, and 7 of conduct disorder. Seventy-seven cases presented with a clinically relevant score $(T>70)$ on the aggression or rule-breaking behavior subscales of the CBCL and 41 cases on both subscales. Thirty-eight cases had an aggression score in the clinical range but no DSM diagnosis (Table 1). While cases and controls were matched for age and handedness, there were more males than females in the case group (99-19); cases also exhibited a lower IQ than healthy controls and showed a wide distribution of RA and PA levels, callous-unemotional traits, and ADHD symptoms. The proportion of controls relative to cases differed across participating sites. For the distribution of diagnoses, aggression scores, medication, and demographic variables by site, please see Supplemental Table S3.

\section{Group differences in functional connectivity}

Compared with controls, cases demonstrated reduced connectivity of the PCC seed with a projection cluster including the left frontal pole $[t(197)=5.46$, cluster-size $p$-FWE $<0.008$, peak uncorrected $p<0.001, \beta=0.10$ ] (Fig. 1, Supplemental Table S4). For the seed in the left anterior insula, after taking into account the ADHD symptoms, cases showed diminished connectivity with a cluster extending from the orbitofrontal cortex $(\mathrm{OFC})$ to the frontal pole $[t(194)=5.07$, cluster-size $p$-FWE $<0.008$, peak uncorrected $p<0.001, \beta=0.10$ ) (Fig. 1, Supplemental Table S4). These analyses were controlled for site. Furthermore, a subsequent analysis revealed a positive correlation 
Table 1 Sample characteristics

\begin{tabular}{|c|c|c|c|c|}
\hline Characteristic & $\begin{array}{l}\text { Cases } \\
(n=118)\end{array}$ & $\begin{array}{l}\mathrm{HC} \\
(n=89)\end{array}$ & Test statistic & $p$ \\
\hline Age (years) & $13.23 \pm 2.68$ & $13.40 \pm 2.49$ & $t(205)=-0.45$ & 0.65 \\
\hline Sex, $m / f$ & $99 / 19$ & $51 / 38$ & $\chi^{2}=17.98$ & $<0.001$ \\
\hline $\mathrm{IQ}^{\mathrm{a}}$ & $100.78 \pm 11.00$ & $106.64 \pm 10.42$ & $t(195)=-3.81$ & $<0.001$ \\
\hline Handedness, left/right & $16 / 95$ & $10 / 77$ & $\chi^{2}=0.37$ & 0.55 \\
\hline CD plus ODD diagnosis ${ }^{b}$ & 25 & & & \\
\hline ODD diagnosis $^{\mathrm{b}}$ & 48 & & & \\
\hline CD diagnosis ${ }^{b}$ & 7 & & & \\
\hline ADHD diagnosis ${ }^{\mathrm{b}}$ & 29 & & & \\
\hline CBCL-Aggression T-score & $74.46 \pm 10.10$ & $51.77 \pm 6.19$ & $t(197)=-12.78$ & $<0.001$ \\
\hline CBCL_-Rule-breaking T-score & $69.00 \pm 12.14$ & $51.69 \pm 6.72$ & $t(196)=-20.54$ & $<0.001$ \\
\hline K-SADS-inattention & $3.33 \pm 2.91$ & & & \\
\hline K-SADS-hyperactivity & $1.66 \pm 1.91$ & & & \\
\hline K-SADS-impulsivity & $1.08 \pm 1.20$ & & & \\
\hline ICU—total score & $33.68 \pm 10.16$ & $21.00 \pm 8.70$ & $t(196)=-9.44$ & $<0.001$ \\
\hline ICU_callousness & $12.00 \pm 6.11$ & $4.00 \pm 3.44$ & $U=2278.00$ & $<0.001$ \\
\hline ICU-uncaring & $17.00 \pm 3.93$ & $10.41 \pm 5.07$ & $U=2445.00$ & $<0.001$ \\
\hline ICU-unemotional & $7.17 \pm 3.31$ & $5.22 \pm 2.75$ & $t(189)=-4.37$ & $<0.001$ \\
\hline $\mathrm{RPQ}$ —reactive aggression & $12.55 \pm 5.09$ & $5.00 \pm 3.48$ & $U=1296.50$ & $<0.001$ \\
\hline $\mathrm{RPQ}$ - proactive aggression & $3.00 \pm 5.01$ & $0.82 \pm 1.45$ & $U=1807.50$ & $<0.001$ \\
\hline Medication use $^{c}$ & 70 & & & \\
\hline Stimulants & 52 & & & \\
\hline Neuroleptics & 18 & & & \\
\hline Antidepressants & 2 & & & \\
\hline Mean RMS-FD & $0.12 \pm 0.17$ & $0.09 \pm 0.18$ & $U=4134.50$ & $<0.01$ \\
\hline
\end{tabular}

Values are means or, for non-normal distributions, medians \pm SD or counts

$A D H D$ attention-deficit hyperactivity disorder, $C B C L$ Child Behavior Checklist, $C D$ conduct disorder, $H C$ healthy controls, $I C U$ inventory of callous-unemotional traits, parent report, $K$-SADS Kiddie Schedule for Affective Disorders and Schizophrenia, $O D D$ oppositional defiant disorder, $R M S-F D$ root mean square framewise displacement, $R P Q$ Reactive and Proactive aggression Questionnaire

${ }^{a}$ IQ estimated based on four sub-tests derived from the Wechsler Intelligence Scale for Children IV

${ }^{b}$ Diagnoses derived from the Kiddie Schedule for Affective Disorders and Schizophrenia Present and Lifetime version

${ }^{\mathrm{c}}$ Medication use according to parental or clinical reports

between ADHD inattention and hyperactivity counts and the strength of connectivity within cases for this left anterior insula-frontal cluster effect, however, at a lower significance threshold $[t(85)>5.27$, all cluster-size $p$-FWE $<0.05$, peak uncorrected $p<0.001, \beta=0.04-0.09$ ] (Fig. 1). There were no other statistically significant findings in these analyses.

\section{Functional connectivity correlates of reactive versus proactive aggression}

Our analysis testing for correlates of RA and PA using the previously applied six seeds yielded subtype-specific results (Fig. 2, Supplemental Table S6). Specifically, PA scores were positively associated with the connectivity strength between the seed in the left amygdala and the precuneal cluster. Moreover, cases with higher RA scores also showed increased connectivity of the seed in the PCC with a cluster extending from the left parahippocampal gyrus to the left inferior temporal gyrus. Furthermore, RA scores were positively correlated with connectivity between the left amygdala seed and a projection cluster extending from the left lateral occipital cortex to the precuneus. There was also a negative association between RA scores and connectivity between the right anterior insula seed and a cluster localized in the right caudate nucleus (all cluster-size $p-\mathrm{FWE}<0.008$, $\beta=0.04-0.05$ ). The above findings remained significant at an uncorrected $p<0.01$ threshold after applying the more stringent excessive motion criterion (see sensitivity analyses, Supplemental Table S11). 

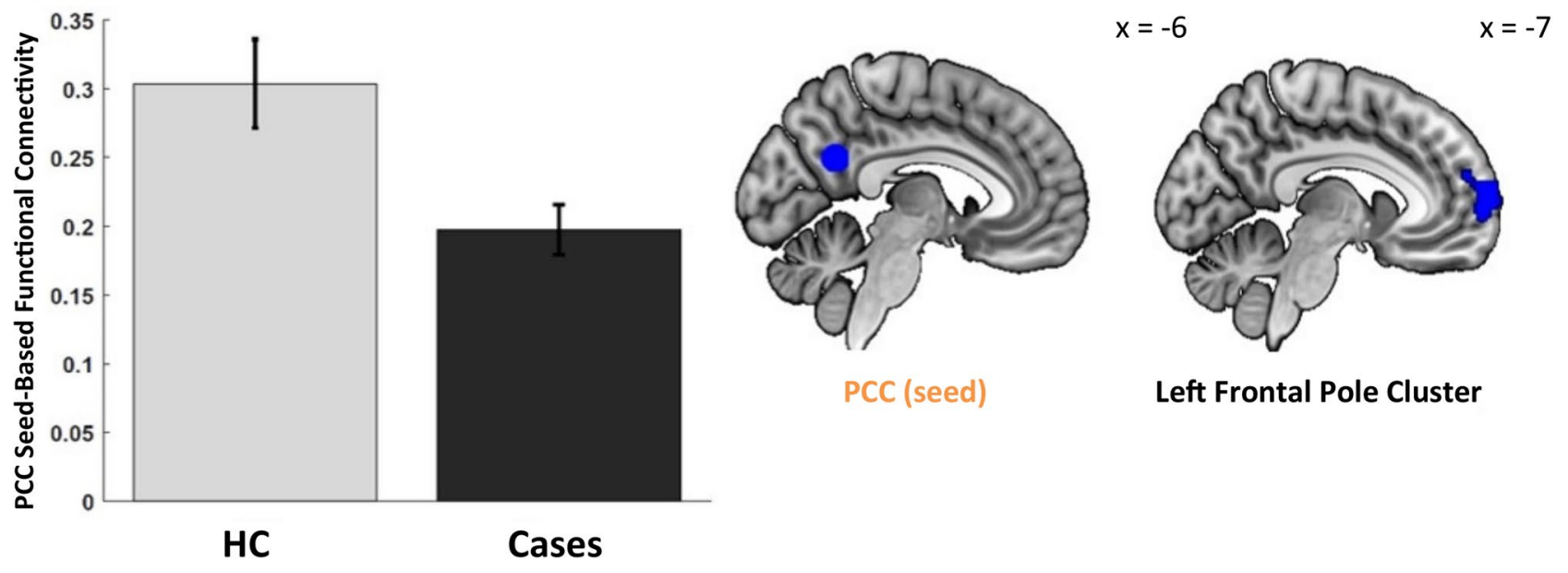

PCC (seed)
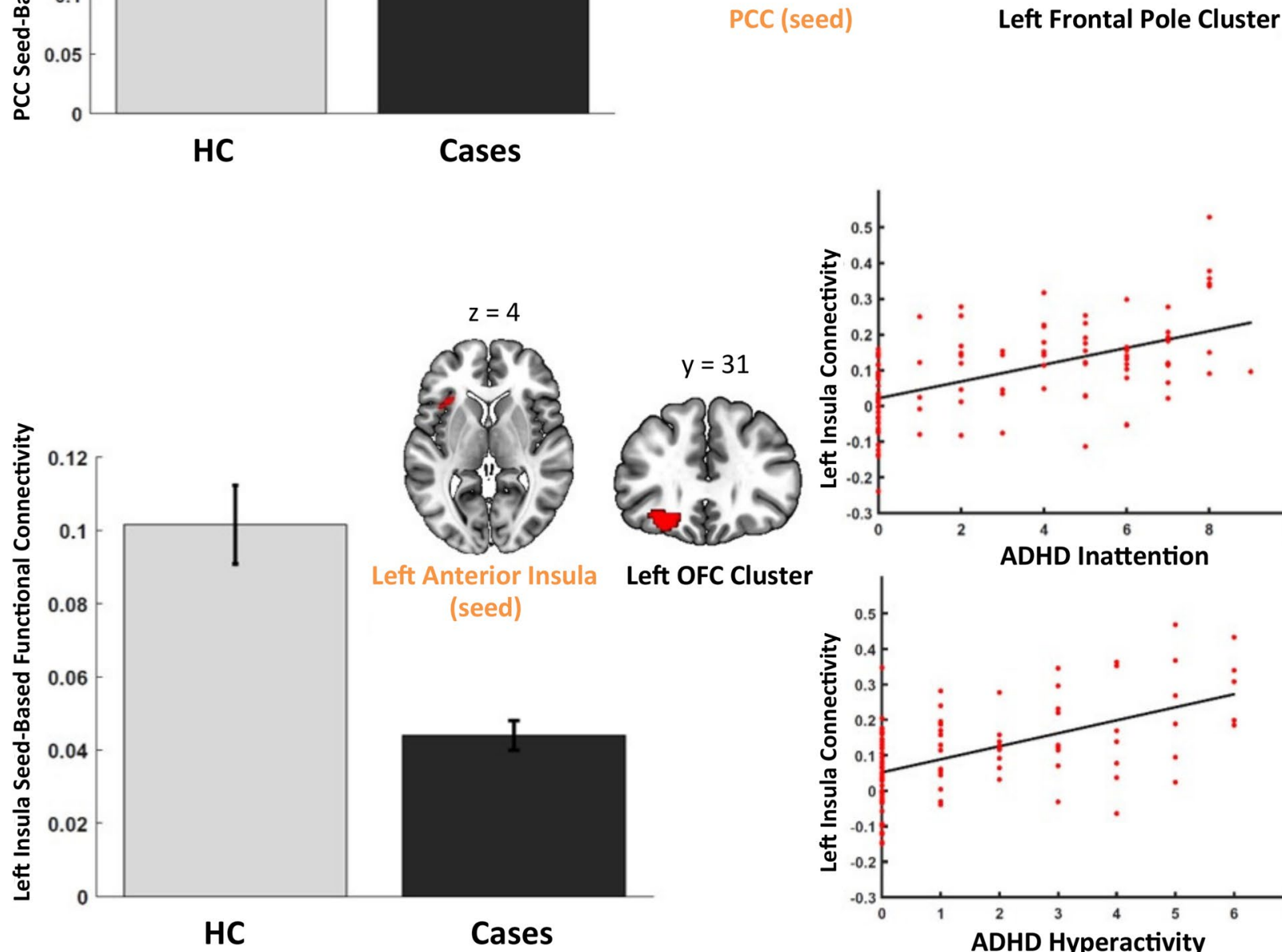

$y=31$
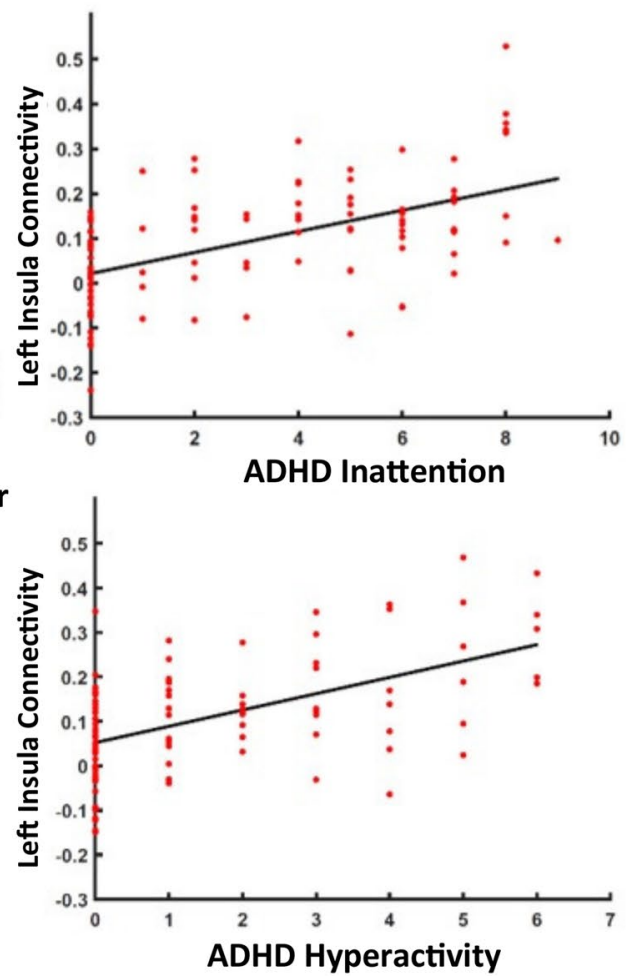

Fig. 1 Significant results from the case-control functional connectivity analysis. The bar charts show a reduced seed-to-voxel connectivity pattern for cases compared to healthy controls (HC). The seeds and corresponding projections are mapped onto a brain diagram. The

\section{Functional connectivity correlates of callous-unemotional traits}

Our analysis testing for correlates of callous-unemotional traits revealed positive associations with distinct seed-based connectivity patterns within cases (Fig. 3, Supplemental Table S7). Specifically, the callousness scores were linked to connectivity between the PCC and a cluster in the right precentral gyrus and cingulate areas, as well as between the

scatterplots depict the main effect of ADHD inattention, and hyperactivity counts in cases plotted against the connectivity values between the seed in the left anterior insula and projection in the left orbitofrontal cluster

amPFC and a region including the right precentral gyrus and precuneus. Uncaring behavior scores were associated with connectivity between the following pairs of regions: the amPFC and right hemispheric cerebellar regions, the left anterior insula and precuneal and cingulate clusters, and the right anterior insula and left central gyrus. Finally, unemotional-specific connectivity was identified for the seed in the left anterior insula and a projection in the precuneus together 


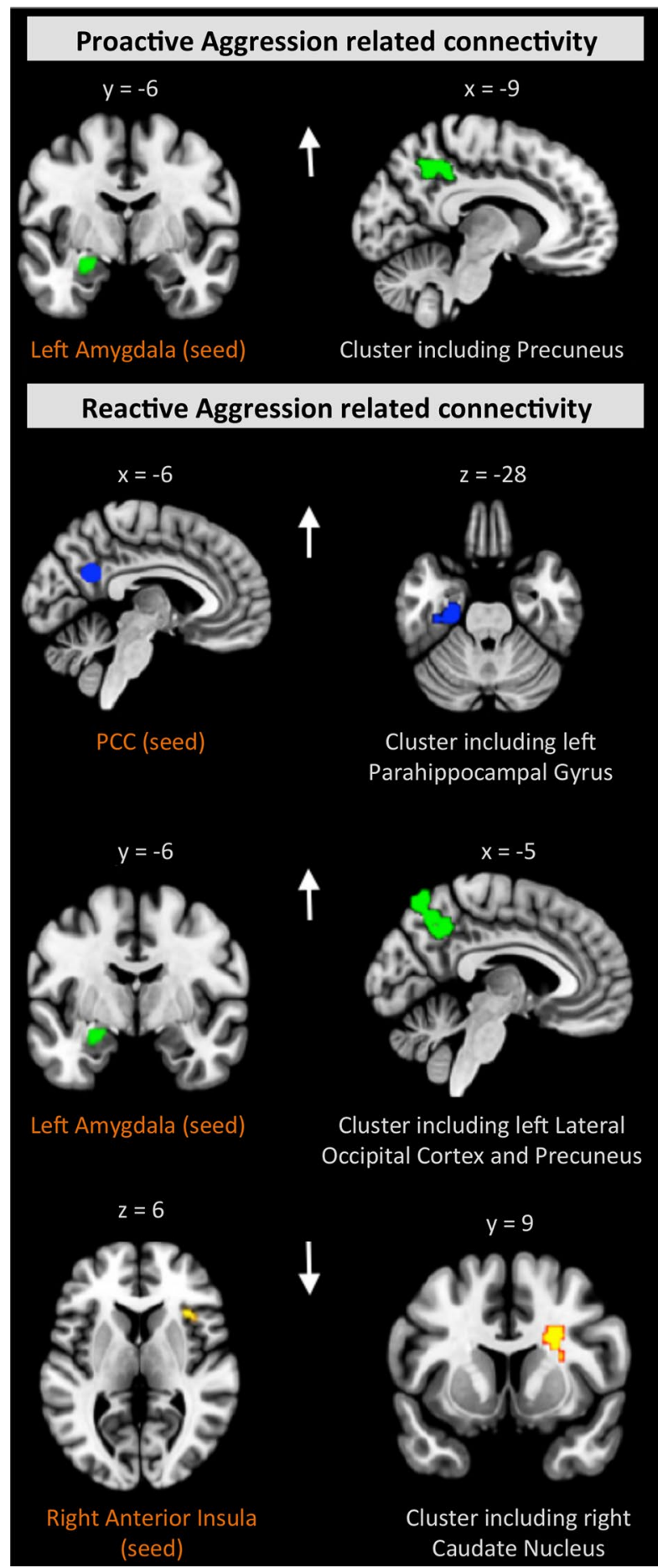

Fig. 2 Distinct RA- and PA-related connectivity patterns within cases. There was only one partly overlapping connectivity pattern related to both RA and PA for the seed in the left amygdala. The seed regions are depicted in the left panel, with corresponding projections shown in the right panel. The results are FWE-corrected, $p<0.008$. The arrows reflect the effect direction (hyper- and hypo-connectivity) with the angular gyrus (all cluster-size $p$-FWE $<0.008$, $\beta=-0.06$ to 0.08 ) (Fig. 3, Supplemental Table S7).

\section{Sensitivity analysis for cases without a DSM diagnosis}

Complementary sensitivity analyses for case-control comparisons were conducted excluding 38 cases without a DSM diagnosis. The results align with the analysis without the exclusion. Cases exhibited a reduced connectivity of the PCC to the left frontal pole $(M=0.20)$ compared to controls $(M=0.30 ; U=3250, p<0.001)$. The effect was present after the exclusion of cases without a diagnosis $(U=2297$, $p<0.001)$, with similar results for cases $(M=0.20)$ and controls $(M=0.30)$. After an additional correction for ADHD scores, cases showed a diminished left anterior insula connectivity $(M=0.05)$ compared to controls $(M=0.10$; $U=2690, p<0.01$ ). Details on all the performed sensitivity analyses are provided in the Supplemental Information.

\section{Effects of covariates on group differences}

For the seed in the PCC, the results withstood controlling for site, age, sex, and IQ at a less conservative significance threshold (cluster-size $p$-FWE $<0.05$ ) and application of a more stringent excessive motion criterion (see Supplemental Table S11 for details on the sensitivity analyses). However, when tested using a full list of covariates, i.e., site, age, sex, IQ, medication, and handedness, no significant results were observed. For the seed in the left anterior insula, the group difference withstood correction for site, age, sex, IQ, and handedness. Additionally, to explore the influence of the covariates as main predictors (sex, IQ, medication, and handedness) on group differences, we conducted sensitivity analyses. These analyses showed that none of these covariates had a significant impact on the relationship between aggression and functional connectivity (all $p>0.05$ ), except as described for the exploratory analyses of age and sex.

\section{Dimensional analysis of the whole sample}

The analysis of functional connectivity correlates in the whole sample showed a decreased connectivity between the PCC and angular gyrus for PA and overlapping increased connectivity of the left amygdala with the precuneus for both PA and RA (Supplementary Table S13-S14, Figure S1), paralleling the effect in cases. 
Fig. 3 Differing connectivity patterns related to scores on the callousness, uncaring, and unemotional dimensions within cases. The seed regions are depicted in the left panel, with corresponding projections shown in the right panel. The results are FWE-corrected, $p<0.008$. The arrows reflect the effect direction (i.e., hyper- and hypo-connectivity)

\section{Age as a moderator}

The dimensional analysis was also rerun with age as a moderator between the connectivity values and clinical scales. Three significant interactions were found only in cases, pointing to increased associations with higher age for the left anterior insula-precuneus and left anterior insula-pre-/ post-central gyri (both for unemotional traits) as well as the right anterior insula-post-central gyri and the total score from the inventory of callous-unemotional traits (see Supplement pages 25-26 and Figure S2 for details).

\section{Exploratory sex-related analyses}

Further, sex by group interactions (after controlling for all other covariates) was found for the connectivity patterns between the left insula and the right superior temporal gyrus. Specifically, female cases showed higher connectivity than female controls, when compared to male participants, who showed lower connectivity in cases than in controls. In the whole sample, there was also lower connectivity in males than females between the amPFC and the superior lateral occipital cortex and between the left insula and the supplementary motor areas and a cluster in the temporal pole. Other effects largely coincided with non-labeled regions (Supplemental Tables S18-S20). There were some tentative sex-dependent effects in relation to the aggression scales and connectivity in cases, being largest for the seed in the right insula and PA and RA (Supplemental Table S21). Sex did not moderate the interaction among the ADHD symptoms (inattention, hyperactivity), group, and connectivity in our main finding (i.e., for the left anterior insula and the left OFC; all $p>0.05)$.

\section{Discussion}

The present multi-center study investigated aggression subtype-specific functional brain connectivity in a sizeable sample of children and adolescents with disruptive behavior, including those with diagnoses of conduct disorder and oppositional defiant disorder. In line with our hypothesis, RA and PA were related to distinct couplings of brain

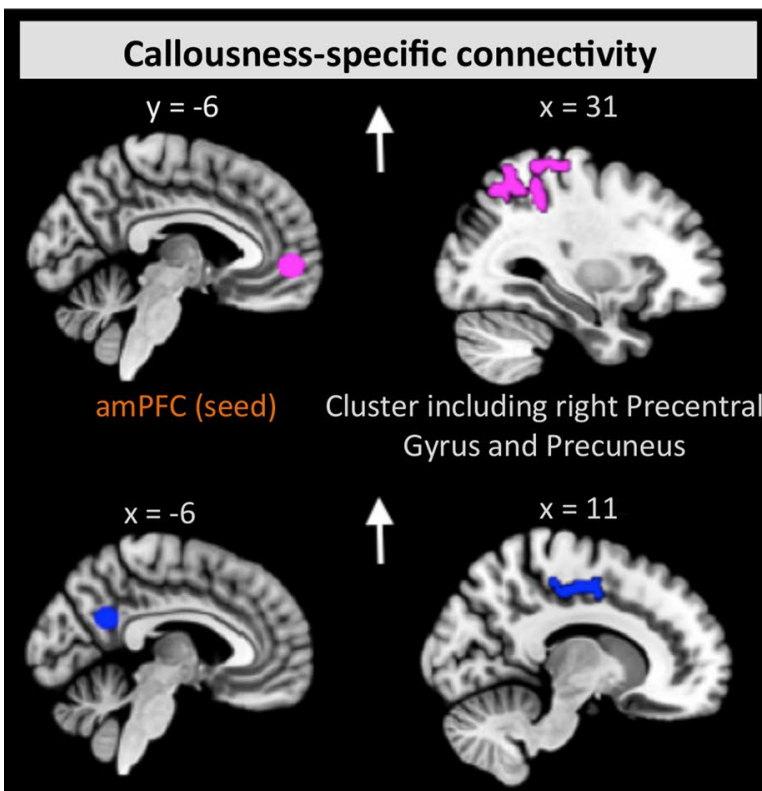

PCC (seed)

Cluster including right Precentral Gyrus and Anterior Cingulate Gyrụs

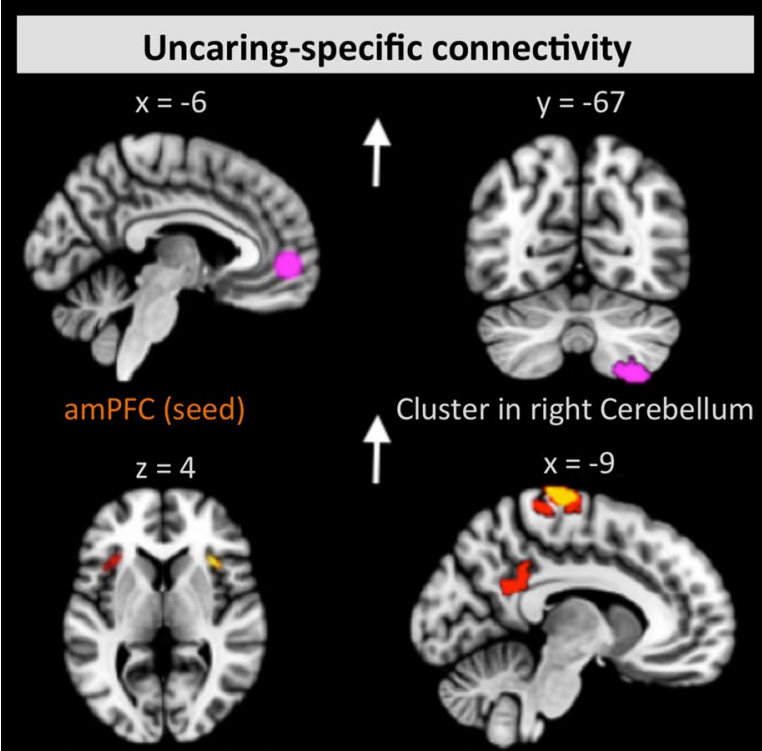

Bilateral Insula (seed)

Cluster including bilateral Central Gyrus and Precuneus

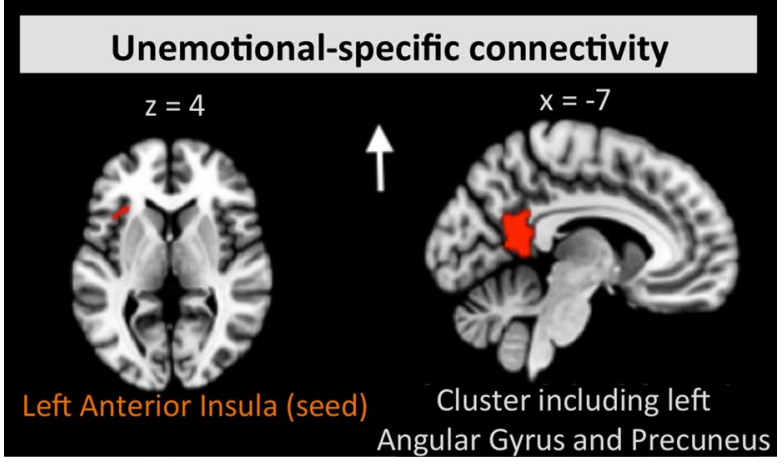


regions implicated in emotion, empathy, and cognitive control. Specifically, PA scores were positively associated with connectivity between the amygdala and precuneus, while RA scores were associated with the increased coupling of the PCC, amygdala, and anterior insula with the (para-)limbic and precuneal clusters. In terms of overlaps, both aggression subtypes involved increased connectivity of the left amygdala with the precuneus. Impaired amygdala function and its connections seem to exert crucial effects on both the neural threat circuitry related to a higher risk for RA and in moral behavior, increasing the risk for PA [47]. The connectivity of the precuneus was closely related to impulsivity in a recent study [24], which represents an important observation given the association of impulsivity and RA [28]. Interestingly, the precuneus cluster in our study was much larger for RA than for PA. Additionally, the amygdala-related projection areas extended to the occipital regions for RA, which may further underpin subtype-specific differentiation, in line with earlier behavioral results [28]. The RA-specific increased connectivity from the PCC to paralimbic and limbic regions aligns with the finding of their abnormal functioning in psychopathy [48]. Specifically, for RA, we found positive functional wiring of the anterior insula with the right caudate nucleus, a region involved with integration of performance and cognitive control [49]. It is known that the neural circuits including the anterior insula regulate the responses to frustration and perceived social provocations [50].

In addition to the PA-/RA-related results, different connectivity patterns were also found for callous-unemotional traits, corroborating previous neural [19, 52, 14, 15, 48] and behavioral findings [52]. As a general remark, our cases exhibited higher connectivity with higher callous-unemotional traits, in the absence of significant negative associations, which is in line with the majority of earlier resting state research literature investigating adolescents [19, 14, 15] and adults [51, 53]. These traits were related in our study to different DMN- and SN-based hyper-connectivities with projection clusters in frontal, parietal, cingulate, precuneal, and cerebellar areas. Specifically, both uncaring and unemotional traits were linked to a stronger coupling between the anterior insula with the precuneal and cingulate clusters, extending to the central and angular gyrus. A recent metaanalytic review confirmed that the right anterior insula and its connectivity are implicated in the evaluation of feelings and the left anterior insula in the expression of anger [54]. Altered insular and cingulate functioning has been previously linked to moral reasoning in adult psychopathy [55]. The results from our study pointed to an association between callousness and uncaring traits and the connectivity patterns involving the precentral gyrus, which has been earlier identified as a hot spot region in adolescents with callous-unemotional traits $[19,18]$ and psychopathic adults $[50,53,56$, 48]. The DMN connectivity effects reported here may also suggest uncaring and callousness dimension-specific alterations in (affective) self-referential processes [43]. Notably, altered connectivity in the DMN has been repeatedly shown in children and adolescents with callous-unemotional traits $[19,18]$ and previously in adult psychopathy $[53,57,58]$. The precuneus, as part of the DMN, contributed to classifying adults with antisocial personality disorder in a previous study [58]. In addition, the overlapping connectivity in the insula projecting to the precuneal and cingulate areas for uncaring and unemotional behavior scores may reflect the variance overlap between both constructs [34]. Intriguingly, we did not find any overlapping connectivity patterns between callous-unemotional traits and RA or PA, despite well-known behavioral commonalities [34, 52] and the behavioral correlation of PA scores with callous-unemotional traits in our sample. Also, contrary to previous reports, no effect on amygdala connectivity was found for these traits $[14,15,59]$. This result aligns with previous findings suggesting neural alterations beyond (para-)limbic regions in adult psychopathy [55]. Interestingly, our most recent study indicated differences in amygdala activity and skin conductance during an emotion-processing task between cases and typically developing controls, with a moderating effect of callous-unemotional traits [60]. Overall, the specific connectivity patterns between seeds and projections identified in the present investigation of callous-unemotional traits, point to mechanisms linked to emotion, empathy, moral, and self-referential processes, which are very often impaired in youth with disruptive behaviors [61].

Compared to healthy controls, cases exhibited diminished connectivity of the PCC (a key brain hub in the DMN) with a cluster in the left frontal pole. By additionally controlling for ADHD symptoms in the analysis, we further identified decreased connectivity between the left anterior insula (a key brain hub of the SN) and a left hemispheric frontal cluster. Abnormal connectivity of the PCC has been demonstrated in male adolescents with conduct disorder [17-19] and may suggest impaired self-referential processes [43]. Our results also support the crucial role of comorbid ADHD symptoms previously shown for the DMN in male adolescents with conduct disorder [17] and extend these findings to the seed-based approach centered in the major DMN and $\mathrm{SN}$ areas with whole-brain projections. In line with previous studies [17, 19, 24], cases exhibited an ADHD scorerelated increase in connectivity, which further corroborates the recent finding of overlapping deficit functioning in ADHD and disruptive behavior disorders [23]. However, the observed group differences only withstood post hoc analysis when other covariates were not controlled for or when a lower statistical threshold was applied. Therefore, a somewhat cautious interpretation of these specific results is merited. 
The present study has some limitations. First, it is worth noting that our 38 aggressive cases without a DSM diagnosis of conduct disorder and/or oppositional defiant disorder exhibited lower PA scores compared to cases with a DSM diagnosis. However, subsequent sensitivity analyses led to comparable results of case-control group comparisons when excluding these cases without a diagnosis. Second, we did not consider the self-reports of callous-unemotional traits. Yet, a recent study suggests a higher criterion validity for parentreported callous-unemotional traits compared to self-reports and teacher reports [62]. Third, the distribution of cases and controls across sites was unbalanced, and both groups were not matched for sex, IQ, or the number of participants. Nevertheless, the sensitivity analyses conducted showed no significant influence of IQ, medication, or handedness on the connectivity of cases compared to controls. Fourth, data for the current study were collected at different sites with equipment from different scanner manufacturers and with partly varying scan parameters, which affected data homogeneity and limited our study power. On the other hand, the larger sample size and the multi-center approach might have increased the reliability and generalizability of our results. Finally, sex-dependent effects were supported by our exploratory analyses, in line with earlier brain imaging studies on aggressive behavior [63]. They should be investigated more in-depth in the future in study samples with comparable sex distributions.

By evaluating the effect of RA and PA along with callousunemotional dimensions, we have extended current knowledge on disruptive behavior disorder and largely understudied distinct manifestations of aggression. These results provide a rationale for treating aggression not as an entity, but as an array of distinct subtypes that can be differentiated on clinical and neural grounds. In particular, therapies for children and adolescents with disruptive behavior may be improved through careful identification of such distinct aggression subtypes, a better understanding of their neural correlates, and the development of interventions counteracting this aberrance. An example of practical application may be realtime fMRI neurofeedback targeting selected brain regions in specific subtypes of aggression and to learn self-regulating brain activity. Moreover, our results may further point to dissociable developmental trajectories, as some of the observed brain areas are also related to adult psychopathy. Notably, RA and PA differentially predict later conduct problems [64], and future studies applying a longitudinal design will be best positioned to track further such effects across time.

Acknowledgements Open access funding provided by University of Zurich. This project has received funding from the European Union's Seventh Framework Program for research, technological development, and demonstration under Grant agreement 602805 (Aggressotype) and no 603016 (MATRICS). This manuscript reflects only the authors' view, and the European Union is not liable for any use that may be made of the information contained herein. MC is currently funded by the Medical Research Council UK (Grant MR/M013588). TB has served in an advisory or consultancy role for Actelion, Hexal Pharma, Lilly, Lundbeck, Medice, Neurim Pharmaceuticals, Novartis, and Shire; he received conference support or speaker's fees from Lilly, Medice, Novartis, and Shire. He has also been involved in clinical trials conducted by Shire and Viforpharma and has received royalties from Hogrefe, Kohlhammer, CIP Medien, and Oxford University Press. CA has been a consultant to or has received honoraria or grants from Acadia, Ambrosseti, Gedeon Richter, Janssen Cilag, Lundbeck, Merck, Otsuka, Roche, Servier, Shire, Schering Plough, Sumitomo Dainippon Pharma, Sunovion, and Takeda. DB serves as an unpaid scientific advisor for an EU-funded neurofeedback trial unrelated to the present work. BF receives funding from a personal Vici grant from the Dutch Organisation for Scientific Research (Grant 016130 669) and the Dutch National Science Agenda for the NWANeurolabNL project (Grant 40017 602). She received educational speaking fees from Shire and Medicine. SW has received lecture honoraria from Lilly and Opopharma/Shire within the last 5 years. She also received royalties from Hogrefe, Kohlhammer, Springer, and Beltz. Outside professional activities and interests are declared on the web page of the University of Zurich (www.uzh.ch/prof/ssl-dir/interessenbindungen/client/web/).

Funding Open access funding provided by University of Zurich.

\section{Compliance with ethical standards}

Conflict of interest The present work is unrelated to the above grants and relationships. The authors do not have potential conflicts of interest. The authors express their gratitude to all participating families and thankfully acknowledge the contributions of Yu Jin Ressel, Noemi Signer, Aline Pfister, Georg Grön, and Kathrin Brändle.

Open Access This article is licensed under a Creative Commons Attribution 4.0 International License, which permits use, sharing, adaptation, distribution and reproduction in any medium or format, as long as you give appropriate credit to the original author(s) and the source, provide a link to the Creative Commons licence, and indicate if changes were made. The images or other third party material in this article are included in the article's Creative Commons licence, unless indicated otherwise in a credit line to the material. If material is not included in the article's Creative Commons licence and your intended use is not permitted by statutory regulation or exceeds the permitted use, you will need to obtain permission directly from the copyright holder. To view a copy of this licence, visit http://creativecommons.org/licenses/by/4.0/.

\section{References}

1. Turgay A (2004) Aggression and disruptive behavior disorders in children and adolescents. Expert Rev Neurother 4(4):623-632

2. Polanczyk GV, Salum GA, Sugaya LS, Caye A, Rohde LA (2015) Annual research review: a meta-analysis of the worldwide prevalence of mental disorders in children and adolescents. J Child Psychol Psychiatry 56(3):345-365

3. American Psychiatric Association (2013) Diagnostic and statistical manual of mental disorders (DSM-5®). American Psychiatric Pub, Washington

4. Frick PJ (2016) Current research on conduct disorder in children and adolescents. S Afr J Psychol 46(2):160-174

5. Essau CA, Sasagawa S, Frick PJ (2006) Callous-unemotional traits in a community sample of adolescents. Assessment 13(4):454-469

6. Blair RJR (2013) The neurobiology of psychopathic traits in youths. Nat Rev Neurosci 14(11):786-799 
7. Dodge KA, Coie JD (1987) Social-information-processing factors in reactive and proactive aggression in children's peer groups. $\mathbf{J}$ Pers Soc Psychol 53(6):1146

8. Daly M (2018) Partitioning aggression. Proc Natl Acad Sci 115(4):633-634

9. Smitha K, Akhil Raja K, Arun K, Rajesh P, Thomas B, Kapilamoorthy $\mathrm{T}$ et al (2017) Resting state fMRI: a review on methods in resting state connectivity analysis and resting state networks. Neuroradiol J 30(4):305-317

10. O'Connor EE, Zeffiro TA (2019) Why is clinical fMRI in a resting state? Front Neurol 10:420

11. Mueller S, Wang D, Fox MD, Yeo BT, Sepulcre J, Sabuncu MR et al (2013) Individual variability in functional connectivity architecture of the human brain. Neuron 77(3):586-595

12. Shen X, Finn ES, Scheinost D, Rosenberg MD, Chun MM, Papademetris X et al (2017) Using connectome-based predictive modeling to predict individual behavior from brain connectivity. Nat Protoc 12(3):506

13. Zhou J, Yao N, Fairchild G, Zhang Y, Wang X (2015) Altered hemodynamic activity in conduct disorder: a resting-state FMRI investigation. PLoS ONE 10(3):e0122750

14. Aghajani M, Colins OF, Klapwijk ET, Veer IM, Andershed H, Popma A et al (2016) Dissociable relations between amygdala subregional networks and psychopathy trait dimensions in conduct-disordered juvenile offenders. Hum Brain Mapp 37(11):4017-4033

15. Aghajani M, Klapwijk ET, van der Wee NJ, Veer IM, Rombouts SA, Boon AE et al (2017) Disorganized amygdala networks in conduct-disordered juvenile offenders with callous-unemotional traits. Biol Psychiat 82(4):283-293

16. Lu F-M, Zhou J-S, Zhang J, Xiang Y-T, Zhang J, Liu Q et al (2015) Functional connectivity estimated from resting-state fMRI reveals selective alterations in male adolescents with pure conduct disorder. PLoS ONE 10(12):e0145668

17. Broulidakis MJ, Fairchild G, Sully K, Blumensath T, Darekar A, Sonuga-Barke EJ (2016) Reduced default mode connectivity in adolescents with conduct disorder. J Am Acad Child Adolesc Psychiatry 55(9):800-08.e1

18. Pu W, Luo Q, Jiang Y, Gao Y, Ming Q, Yao S (2017) Alterations of brain functional architecture associated with psychopathic traits in male adolescents with conduct disorder. Sci Rep 7(1):1-11

19. Cohn MD, Pape LE, Schmaal L, van den Brink W, van Wingen G, Vermeiren RR et al (2015) Differential relations between juvenile psychopathic traits and resting state network connectivity. Hum Brain Mapp 36(6):2396-2405

20. Thijssen S, Kiehl KA (2017) Functional connectivity in incarcerated male adolescents with psychopathic traits. Psychiatry Res Neuroimaging 265:35-44

21. Waschbusch DA (2002) A meta-analytic examination of comorbid hyperactive-impulsive-attention problems and conduct problems. Psychol Bull 128(1):118

22. Hawes DJ, Dadds MR (2005) The treatment of conduct problems in children with callous-unemotional traits. J Consult Clin Psychol 73(4):737

23. Puiu A, Wudarczyk O, Goerlich K, Votinov M, Herpertz-Dahlmann B, Turetsky B et al (2018) Neuroscience and Biobehavioral Reviews Impulsive aggression and response inhibition in attention-deficit/hyperactivity disorder and disruptive behavioral disorders: findings from a systematic review. Neurosci Biobehav Rev 90:231-246

24. Lu F-M, Zhou J-S, Wang X-P, Xiang Y-T, Yuan Z (2017) Short-and long-range functional connectivity density alterations in adolescents with pure conduct disorder at resting-state. Neuroscience 351:96-107

25. Lu F-M, Zhou J-S, Zhang J, Wang X-P, Yuan Z (2017) Disrupted small-world brain network topology in pure conduct disorder. Oncotarget 8(39):65506
26. Uytun MC, Karakaya E, Oztop DB, Gengec S, Gumus K, Ozmen $S$ et al (2017) Default mode network activity and neuropsychological profile in male children and adolescents with attention deficit hyperactivity disorder and conduct disorder. Brain Imaging Behav 11(6): 1561-1570

27. Polman H, de Castro BO, Koops W, van Boxtel HW, Merk WW (2007) A meta-analysis of the distinction between reactive and proactive aggression in children and adolescents. J Abnorm Child Psychol 35(4):522-535

28. Fite PJ, Stoppelbein L, Greening L (2009) Proactive and reactive aggression in a child psychiatric inpatient population: Relations to psychopathic characteristics. Crim Justice Behav 36:481-493

29. Zhou J, Yao N, Fairchild G, Cao X, Zhang Y, Xiang Y-T et al (2016) Disrupted default mode network connectivity in male adolescents with conduct disorder. Brain Imaging Behav 10(4):995-1003

30. Achenbach TM (1991) Integrative guide for the $1991 \mathrm{CBCL} / 4-18$, YSR, and TRFprofiles. University of Vermont, Department of Psychiatry, Burlington, VT

31. Muehlhan M, Lueken U, Wittchen H-U, Kirschbaum C (2011) The scanner as a stressor: evidence from subjective and neuroendocrine stress parameters in the time course of a functional magnetic resonance imaging session. Int J Psychophysiol 79(2):118-126

32. Kaufman J, Birmaher B, Brent D, Rao U, Flynn C, Moreci P et al (1997) Schedule for affective disorders and schizophrenia for school-age children-present and lifetime version (K-SADSPL): initial reliability and validity data. J Am Acad Child Adolesc Psychiatry 36(7):980-988

33. Raine A, Dodge K, Loeber R, Gatzke-Kopp L, Lynam D, Reynolds $C$ et al (2006) The reactive-proactive aggression questionnaire: differential correlates of reactive and proactive aggression in adolescent boys. Aggress Behav 32(2):159-171

34. Kimonis ER, Frick PJ, Skeem JL, Marsee MA, Cruise K, Munoz LC et al (2008) Assessing callous-unemotional traits in adolescent offenders: validation of the inventory of callous-unemotional traits. Int J Law Psychiatry 31(3):241-252

35. Christoff K, Irving ZC, Fox KC, Spreng RN, Andrews-Hanna JR (2016) Mind-wandering as spontaneous thought: a dynamic framework. Nat Rev Neurosci 17(11):718

36. Calhoun VD, Wager TD, Krishnan A, Rosch KS, Seymour KE, Nebel MB et al (2017) The impact of T1 versus EPI spatial normalization templates for fMRI data analyses. Hum Brain Mapp 38(11):5331-5342

37. Behzadi Y, Restom K, Liau J, Liu TT (2007) A component based noise correction method (CompCor) for BOLD and perfusion based fMRI. Neuroimage 37(1):90-101

38. Whitfield-Gabrieli S, Nieto-Castanon A (2012) Conn: a functional connectivity toolbox for correlated and anticorrelated brain networks. Brain Connect 2(3):125-141

39. Chai XJ, Castañón AN, Ongür D, Whitfield-Gabrieli S (2012) Anticorrelations in resting state networks without global signal regression. Neuroimage 59(2):1420-1428

40. Power JD, Barnes KA, Snyder AZ, Schlaggar BL, Petersen SE (2012) Spurious but systematic correlations in functional connectivity MRI networks arise from subject motion. Neuroimage 59(3):2142-2154

41. Von Rhein D, Oldehinkel M, Beckmann CF, Oosterlaan J, Heslenfeld D, Hartman CA et al (2016) Aberrant local striatal functional connectivity in attention-deficit/hyperactivity disorder. J Child Psychol Psychiatry 57(6):697-705

42. Jacobs R, Barba A, Gowins J, Klumpp H, Jenkins L, Mickey B et al (2016) Decoupling of the amygdala to other salience network regions in adolescent-onset recurrent major depressive disorder. Psychol Med 46(5):1055-1067 
43. Andrews-Hanna JR, Reidler JS, Sepulcre J, Poulin R, Buckner RL (2010) Functional-anatomic fractionation of the brain's default network. Neuron 65(4):550-562

44. Holz NE, Boecker-Schlier R, Buchmann AF, Blomeyer D, Jennen-Steinmetz C, Baumeister S et al (2017) Ventral striatum and amygdala activity as convergence sites for early adversity and conduct disorder. Soc Cognit Affect Neurosci 12(2):261-272

45. Gasquoine PG (2014) Contributions of the insula to cognition and emotion. Neuropsychol Rev 24(2):77-87

46. Mak LE, Minuzzi L, MacQueen G, Hall G, Kennedy SH, Milev $R$ (2017) The default mode network in healthy individuals: a systematic review and meta-analysis. Brain Connect 7(1):25-33

47. Blair RJR (2010) Neuroimaging of psychopathy and antisocial behavior: a targeted review. Curr Psychiatry Rep 12(1):76-82

48. Espinoza FA, Vergara VM, Reyes D, Anderson NE, Harenski CL, Decety J et al (2018) Aberrant functional network connectivity in psychopathy from a large $(\mathrm{N}=985)$ forensic sample. Hum Brain Mapp 39(6):2624-2634

49. Brovelli A, Nazarian B, Meunier M, Boussaoud D (2011) Differential roles of caudate nucleus and putamen during instrumental learning. Neuroimage 57(4):1580-1590

50. Blair RJ (2016) The neurobiology of impulsive aggression. J Child Adolesc Psychopharmacol 26(1):4-9

51. Philippi CL, Pujara MS, Motzkin JC, Newman J, Kiehl KA, Koenigs M (2015) Altered resting-state functional connectivity in cortical networks in psychopathy. J Neurosci 35(15):6068-6078

52. Pechorro P, Ray JV, GonÇalves RA, Jesus SN (2017) The inventory of callous-unemotional traits: psychometric properties among referred and non-referred Portuguese female juveniles. Int J Law Psychiatry 54:67-75

53. Tang Y, Long J, Wang W, Liao J, Xie H, Zhao G et al (2016) Aberrant functional brain connectome in people with antisocial personality disorder. Sci Rep 6:26209

54. Lindquist KA, Wager TD, Kober H, Bliss-Moreau E, Barrett LF (2012) The brain basis of emotion: a meta-analytic review. Behav Brain Sci 35(3):121
55. Griffiths SY, Jalava JV (2017) A comprehensive neuroimaging review of PCL-R defined psychopathy. Aggress Violent Behav 36:60-75

56. Korponay C, Pujara M, Deming P, Philippi C, Decety J, Kosson DS et al (2017) Impulsive-antisocial dimension of psychopathy linked to enlargement and abnormal functional connectivity of the striatum. Biol Psychiatry 2(2):149-157

57. Pujol J, Batalla I, Contreras-Rodríguez O, Harrison BJ, Pera V, Hernández-Ribas R et al (2012) Breakdown in the brain network subserving moral judgment in criminal psychopathy. Soc Cognit Affect Neurosci 7(8):917-923

58. Tang Y, Jiang W, Liao J, Wang W, Luo A (2013) Identifying individuals with antisocial personality disorder using resting-state FMRI. PLoS ONE 8(4):e60652

59. Salekin RT (2017) Research review: what do we know about psychopathic traits in children? J Child Psychol Psychiatry 58(11):1180-1200

60. Aggensteiner P-M, Holz NE, Böttinger BW, Baumeister S, Hohmann S, Werhahn JE et al (2020) The effects of callous-unemotional traits and aggression subtypes on amygdala activity in response to negative faces. Psychol Med. https://doi.org/10.1017/S003329172 0002111

61. Blair R, Veroude K, Buitelaar J (2018) Neuro-cognitive system dysfunction and symptom sets: a review of fMRI studies in youth with conduct problems. Neurosci Biobehav Rev 91:69-90

62. Docherty M, Boxer P, Huesmann LR, O’Brien M, Bushman B (2017) Assessing callous-unemotional traits in adolescents: determining cutoff scores for the inventory of callous and unemotional traits. J Clin Psychol 73(3):257-278

63. Rogers JC, Gonzalez-Madruga K, Kohls G, Baker RH, Clanton RL, Pauli R et al (2019) White matter microstructure in youths with conduct disorder: effects of sex and variation in callous traits. J Am Acad Child Adolesc Psychiatry 58(12):1184-1196

64. Vitaro F, Gendreau PL, Tremblay RE, Oligny P (1998) Reactive and proactive aggression differentially predict later conduct problems. J Child Psychol Psychiatry 39(3):377-385

\title{
Affiliations
}

\author{
Julia E. Werhahn ${ }^{1,2}$ (1) - Susanna Mohl ${ }^{1}$ - David Willinger ${ }^{1,2}$ - Lukasz Smigielski ${ }^{1}$ - Alexander Roth ${ }^{1,2}$. \\ Christoph Hofstetter ${ }^{1}$. Philipp Stämpfli ${ }^{3}$. Jilly Naaijen ${ }^{4,5}$. Leandra M. Mulder ${ }^{4,5}$. Jeffrey C. Glennon ${ }^{4}$. \\ Pieter J. Hoekstra ${ }^{6} \cdot$ Andrea Dietrich $^{6} \cdot$ Renee Kleine Deters $^{6} \cdot$ Pascal M. Aggensteiner $^{7} \cdot$ Nathalie E. Holz $^{7}$. \\ Sarah Baumeister ${ }^{7}$. Tobias Banaschewski ${ }^{7}$. Melanie C. Saam ${ }^{8}$. Ulrike M. E. Schulze ${ }^{8}$. David J. Lythgoe ${ }^{9}$. \\ Arjun Sethi ${ }^{10}$. Michael C. Craig ${ }^{10}$. Mathilde Mastroianni ${ }^{11}$ - Ilyas Sagar-Ouriaghli ${ }^{11}$. Paramala J. Santosh ${ }^{11}$. \\ Mireia Rosa ${ }^{12}$. Nuria Bargallo ${ }^{13}$. Josefina Castro-Fornieles ${ }^{14}$. Celso Arango ${ }^{15}$. Maria J. Penzol ${ }^{15}$. Marcel P. Zwiers ${ }^{5}$. \\ Barbara Franke $^{16,17}$. Jan K. Buitelaar ${ }^{4,18}$. Susanne Walitza ${ }^{1,2}$. Daniel Brandeis ${ }^{1,2,7}$
}

1 Department of Child and Adolescent Psychiatry and Psychotherapy, University Hospital of Psychiatry, University of Zurich, Neumünsterallee 9, 8032 Zurich, Switzerland

2 Neuroscience Center Zurich, University of Zurich and ETH Zurich, Zurich, Switzerland

3 Department of Psychiatry, Psychotherapy and Psychosomatics and Department of Child and Adolescent Psychiatry, Psychiatric Hospital, MR-Center, University of Zurich, Zurich, Switzerland

4 Donders Institute for Brain, Cognition and Behaviour, Department of Cognitive Neuroscience, Radboud University Medical Center, Nijmegen, The Netherlands
5 Donders Institute for Brain, Cognition and Behaviour, Centre for Cognitive Neuroimaging, Radboud University, Nijmegen, The Netherlands

6 Department of Child and Adolescent Psychiatry, University of Groningen, University Medical Center Groningen, Groningen, The Netherlands

7 Department of Child and Adolescent Psychiatry and Psychotherapy, Central Institute of Mental Health, Medical Faculty Mannheim/ Heidelberg University, Mannheim, Germany

8 Department of Child and Adolescent Psychiatry/Psychotherapy, University Hospital, University of Ulm, Ulm, Germany 
9 Department of Neuroimaging, Institute of Psychiatry, Psychology and Neuroscience, King's College London, London, UK

10 Department of Forensic and Neurodevelopmental Sciences, Institute of Psychiatry, Psychology and Neuroscience, King's College London, London, UK

11 Department of Child Psychiatry, Institute of Psychiatry, Psychology and Neuroscience, King's College London, London, UK

12 Child and Adolescent Psychiatry Department, Hospital Clinic of Barcelona, IDIBAPS, Barcelona, Spain

13 Clinic Image Diagnostic Center (CDIC), Hospital Clinic of Barcelona, Magnetic Resonance Image Core Facility, IDIBAPS, Barcelona, Spain

14 Child and Adolescent Psychiatry and Psychology Department, Institute Clinic of Neurosciences, Hospital Clinic of Barcelona, CIBERSAM, IDIBAPS, Department of Medicine, University of Barcelona, Barcelona, Spain
15 Child and Adolescent Psychiatry Department, Hospital General Universitario Gregorio Marañón School of Medicine, IiSGM, CIBERSAM, Universidad Complutense, Madrid, Spain

16 Department of Human Genetics, Donders Institute for Brain, Cognition and Behaviour, Radboud University Medical Center, Nijmegen, The Netherlands

17 Department of Psychiatry, Donders Institute for Brain, Cognition and Behaviour, Radboud University Medical Center, Nijmegen, The Netherlands

18 Karakter Child and Adolescent Psychiatry University Center, Nijmegen, The Netherlands 